\title{
Smart Collar and Chest Strap Design for Rescue Dog through Multidisciplinary Approach
}

\author{
Fang-Lin Chao ${ }^{1}$, Wei Zhong Feng ${ }^{2, *}$, Kaiquan $\mathrm{Shi}^{1}$ \\ ${ }^{1}$ Department of Industrial Design, Chaoyang University of Technology, Taichung, 436, Taiwan \\ ${ }^{2}$ Department of Visual Communication, Chaoyang University of Technology, Taichung, 436, Taiwan
}

\begin{tabular}{l} 
A R T I C L E I N F O \\
\hline Article history: \\
Received: 09 November, 2020 \\
Accepted: 13 January, 2021 \\
Online: 22 January, 2021 \\
\hline
\end{tabular}

Keywords:

Rescue dog

IoT device

Concept design

Remote interaction

Design education

\begin{abstract}
A B S T R A C T
Rescuers escorted search dogs into the disaster area, using their unique sense of smell to find the injured. First, researchers summarize the design requirements in the search process from interviews with rescuers, and construct a conceptual prototype to confirm the interaction mode between the user and the dog. User central design invited people melt into the situation to identify product features. The ideas were selected based on the viability which increases efficiency. The main design proposal includes a strap and a smart collar. Smart sensing (heartbeat, speed, temperature, and GPS) can improve communication and increases the efficiency of rescue. The search area is large in many cases; therefore, we selected the WiFi or Ultra-wideband module as the wireless transmission medium when the rescue team enters this domain. The pre-deploy nodes connect and position with the smart collars. The instructor sends voice commands remotely to prompt the dog to return when the temperature is high. The smart collar design includes an elastic O-ring waterproof shell. Rescuers click the recall button, and the remote device sends a signal of dog returning. This proposed work looks more at user's needs through multi-disciplinary aspects of view, which enhance usability. The case consists of customer interviews, observation, concepts, evaluation (sciencel devicel electronic packaging/ and App software); the design process also demonstrated a possible teamwork perspective in the industry. This scenario encourages cross-field extension for design education.
\end{abstract}

\section{Introduction}

Rescue dogs are sensitive to hearing and smell [1], and can be used as rubble rescue dogs and mountain rescue dogs after personalized training. This research proposed suitable devices for rescue task. During task, dog will present a stabilize bark to mark the sufferer position and special events [2]. The rescue team fights for gold rescue time to locate suffered people in the mountains or landslides space. Inappropriate equipment reduce efficiency.

The design goal is improving communication efficiency of the training with the help of smart collar through the design process (session 3). The design team collected requirements (session 3.1) through user interviews (session 2.1); after the midterm meeting, we built the prototype (session 4.1, 4.2) to support the interaction between the user and the dog. From the observation of the rescue member's situation, we confirm the rationality of the design (session 4.4, 4.5).

*Corresponding Author: W.Z. Feng, 79 Futong St., Taichung 436, Taiwan R.O.C., 886-918276088, feng.jimmy@msa.hinet.net

\section{Literature survey}

\subsection{Rescue dog training situation and facilities}

Figure 1a shows the rescue dog training cite in central Taiwan, the site is suitable for air scent search of the surrounding or trace the odors. Figure 1b is layout and facilities in Taichung training area. Trainer evaluate dogs and determine working potential to give the dog of their skills to fulfilling its purpose.

The training ground provides olfactory practice and obstacle crossing in a large area, and then enters the building of the injured person through overcome obstacles, such as suspended bridge structure. Simulation provides robustness under various conditions of the dog. Dogs in rocky areas need to be careful not to hurt their paws when moving (Figure 2). The training included the communication skill with the rescue personnel to notify the location of the victim [2]. When the road is in poor condition, the foot is often injured. Dogs often stick out their tongues to strengthen sweat; long-term search can lead to body failure [3]. Passing on the current physical condition allows calling for retreat. 


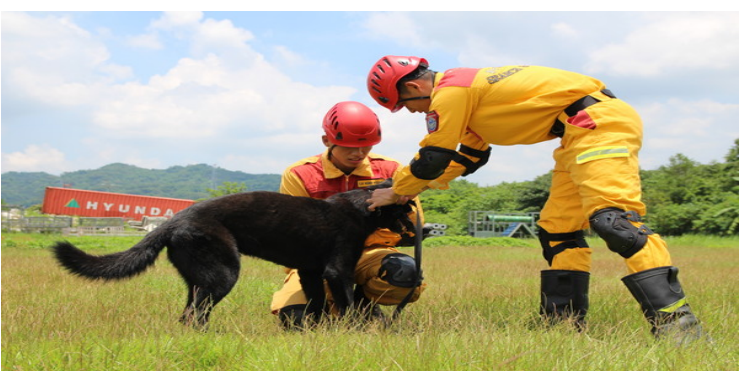

(a)

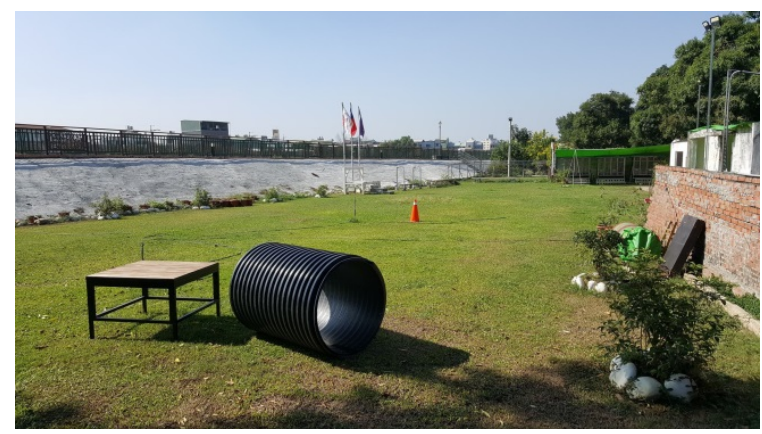

(b)

Figure 1: Rescue dog training in Taiwan: (a) field survey site; (b) Taichung training area.

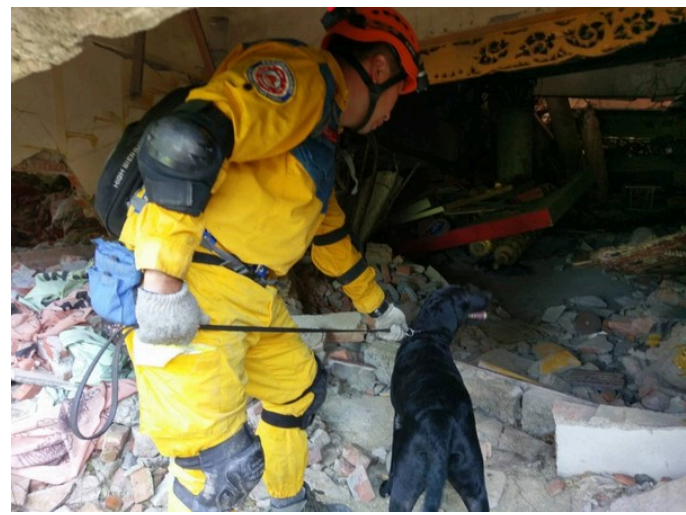

Figure 2: Training situation and facilities in rocky area.

\subsection{Prior art of Strap}

There are several ideas of dog lash as follows: Double D-ring rope buckle, LED warning; H-shaped chest for quick-fitting; Xshaped design using breathable fabric, and Y-shaped structure on the front chest. Double leash keep safe of manage reactive dog with a front and back ring. A leash shifts the center of gravity back towards trainer which gives trainer a pivot point [4] without restricting dog's shoulder for freely searching. Dog is dependent on trainer to explore the sights, trainer have to teach "how to follow lead" or teaching commands through strap.

Sokolowski explored 3D hand grip shape for dog walking which captured from civilian's [5] to fulfill product design perspective. Both cognitive and physical abilities help a successful handler design [6]. Figure 3 reveals an ergonomic X-strap dog harness. The traction belt is smooth and evenly bears multidirectional forces. Another design is an enhanced retractable leash [7] which provides multiple handles for controlling the movement. Plastic buckles are utilized with Velcro straps which eliminate the leash pulling forces at the dog's center of mass (middle chest) area.
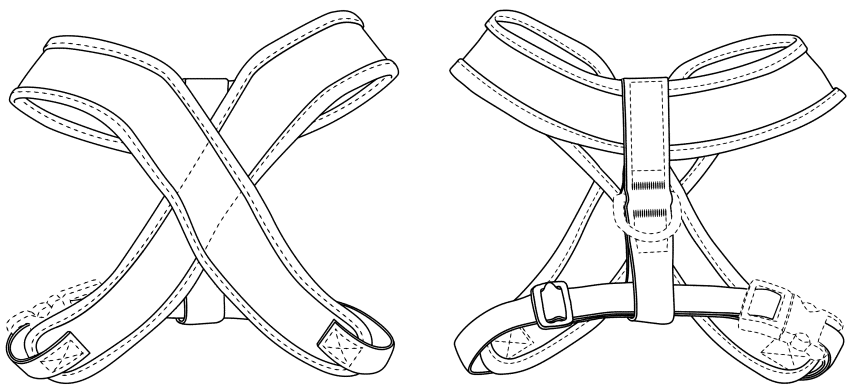

Figure 3: Ergonomic design X strap for dog, US 29/395,500 [6].

\subsection{Scent and airflow}

The skin disseminates cells with special odors in the metabolism, which form diffusion due to air convection. When dogs approach, they can feel the odor; after being trained in specific odors, it can increase sensitivity [2]. During the training process, the dog tracked across the sidewalk, turf, and forest path; the notification after sniffing helps people confirm the rescue path.

Researchers [3] developed a two-part dog wearable computer and interface. In the concept design process, the team first interviewed experts to correctly explain the dog's behavior. When the dog finds impure odor sources, timely encouragement by the trainer can improve its ability [8]. The experiment determined the distance and concentration that the dog can recognize, and was surprised to find that the dog can detect the smell at a distance of more than 62 meters.

\subsection{IoT device in dog/human interaction}

The elderly-animal friendship bond surveyed elf-perceived criteria by the old people regarding their intimate association with their dogs [9]. A survey of 60 people $[10,11]$ asked people classify the behavior of the nine dogs in the video as "friendly" or "radical." Researchers found that the dog's tail's movement is the main clue to explain the dog's sensation $[12,13]$.

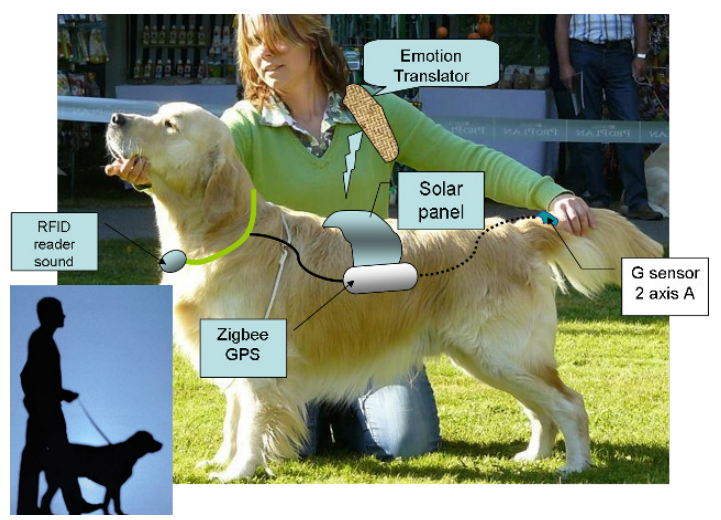

Figure 4: Placement of the major components and main controller [15]

Sensor and actuator help the communication between the blind and the dog; the power spectral density detection method based on breathing sound discovers the physiological state related to different odor detection tasks [14]. Previous study [15] proposed a remote interaction device implemented with Zigbee wireless technology between pet and elderly. The signal provided outdoors activities monitoring and communication, the module placed on 
the back of the dog, and issue commands using a voice pager. The control chassis consist of battery, charger circuit and Zigbee circuit (Figure 4). Owner use vibrating actuator to command the dog remotely. Other study developed [16] an algorithm for locating victims in the nearly collapsed building with indoors positioning and extra IR and sound sensors.

\subsection{Possibility of WiFi nodes}

Many rescue areas do not present a WiFi connection. If WiFi is not available, the trainer depends on the sound to communicate. The mobile station could install, which provides the possibility of access in an emergency. Moreover, Ultra-wideband (UWB) and Long Range (LoRa) were utilized for a high-precision UAV positioning [17] with altitude error reduced to $1.74 \mathrm{~m}$. Simultaneous localization could be obtained through WiFi signal strength measurements [18]. Received signal strength data provide a convent way of practical implementation with a set of nodes.

The above technology is accurate, but this study focuses on the interaction between the trainer and the dog. To simplify the complexity, we use the WiFi module for the usability evaluation.

\section{Design Method}

The successful design of a product takes into account the user response, the alternative designs can amplify designers' understanding of the intended purpose [19]. The user central design reduced error and accelerating the decision-making process [20].

\subsection{Design process}

We collected user desire through interviews with rescue team. Organize their feelings into group records and use the data as a source of concept design. A scenario invited people melt into the pre-settled situation and identified needs. The conceptual ideas were evaluated based on the viable characteristics. The design flowchart shows in Figure 5.

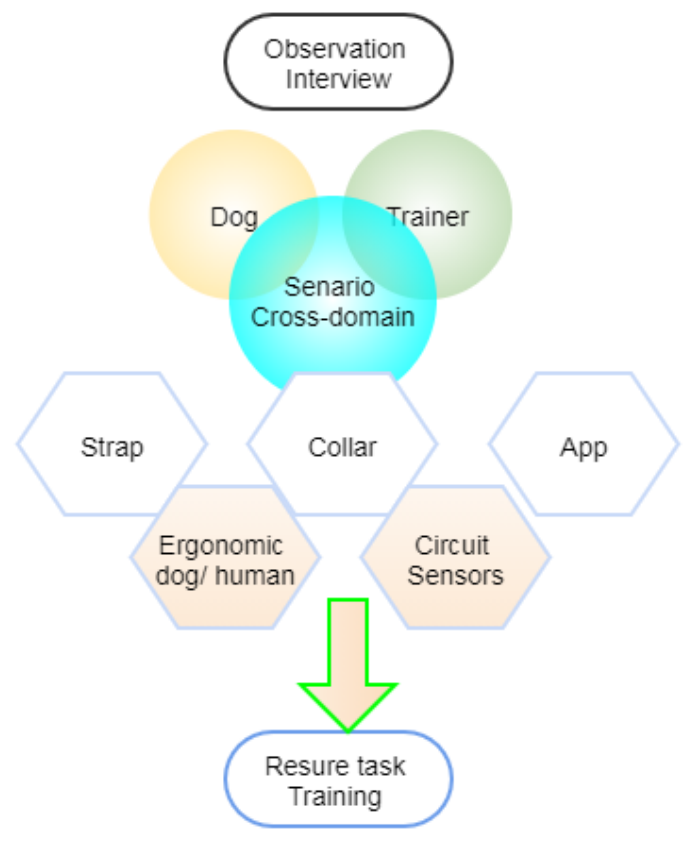

Figure 5: The design process and flowchart
Inquiring with professionals from the Taichung rescue team, the researcher also participated volunteer activity to observe the training details. Interview data shows: dog looks down and scans left and right for evidence. When the trainer pulls back, the neck will be red and swollen. An inappropriate chest strap will cause friction and injury to the fur. Don't hinder neck movement; prevent objects from hooking; use breathable materials when hot and humid.

The design begins with the scenario which deduced from insight of the task and following directions were issued;

1) Include small flashlights and emergency relief kits, but do not hinder neck movement.

2) The disaster may face rainy environmental and need waterproof capability.

3) Taiwan is hot and humid, and dogs do not dissipate heat well. Breathable and thin materials is preferred.

4) GPS-path records located the trapped; the App is needed to improve the efficiency of search.

5) Health datas (dog's heartbeat and body condition) are essential for physical condition control.

Figure 6 shows the selected design concepts of a tracking device and straps which reduces the obstacles interference during the mission. The design features overcome the current product are following:

1) Does not hinder the search activities. When the dog looks down, the branches may get caught in the leash or traction loop and be injured.

2) The product frame is breathable, avoiding heat stroke during dog wearing, which satisfied design priority of: comfort, safety and dog's acceptance. The strap disperses pulling forces on the muscles.

3) Dogs and rescuers can support each other through complementarity, but the configuration of equipment cannot interfere with the task.

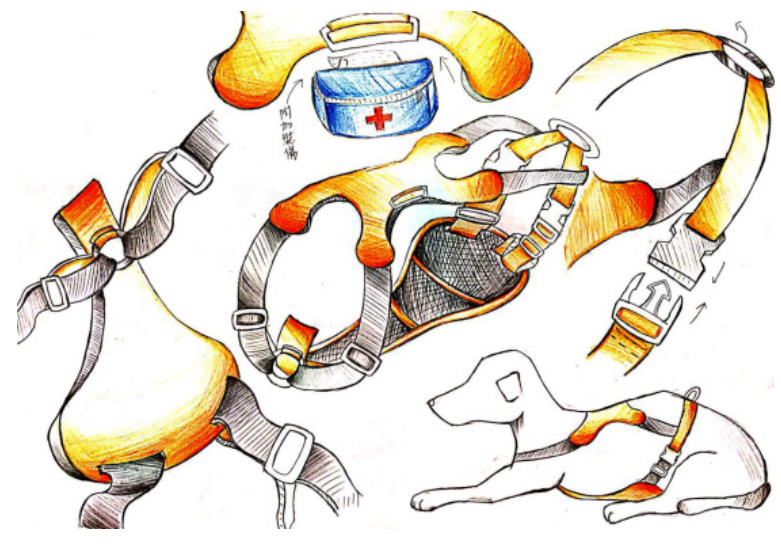

Figure 6: The idea sketch of design concept

\subsection{Multidisciplinary approach}

The implementation requires a cross-domain integration by running a multidisciplinary collaborative project. The ability to lead a team through the different work stages of a project is a fundamental contributor to its success [21]. Framework for the conceptual design of unmanned aerial vehicles (UAVs) proposed 
in developing efficient winged design [22] for industrial designer. Teachers used cross-domain design and developed " airflow play with music" to realize the concept of flow guiding.

Comfort rescue dogs' products needs structural mechanics, material applications, and most importantly, software and hardware integration. Cross-domain presentation requires communication and interaction between departments. The conceptual designer proposes idea sketch and functions supported in the context of use, which discussed with the engineer in advance. Finally, it can be realized with appropriate technology. This process includes the assembly of electronic circuit boards, and software and hardware test to present the design concepts. Then through the deduction of the concept the design verified with the cross-domain team.

\section{Concept design}

\subsection{Chest strap}

Comfortable shoulder straps need to be worn quickly and fit the body without friction. The chest $\mathrm{H}$-shaped backrest equipped with an adjustable double belt buckle. The Y-shaped structure made of breathable fabrics reduces tensile strength (nylon, plastic and fluorescent reflective tape). The front stainless steel O-ring can release and reduce pressure.

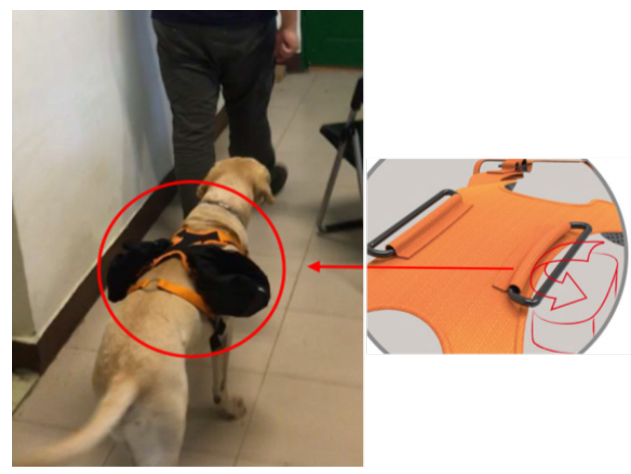

(a)

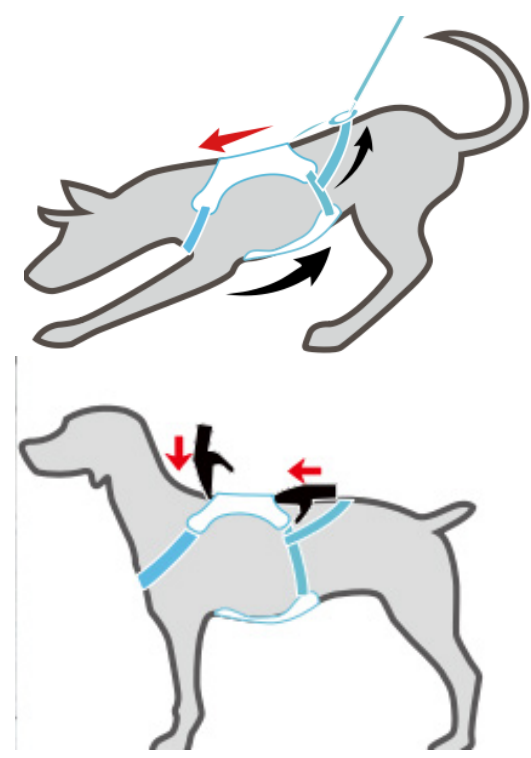

(b)

Figure 7: (a) the upper chest pad and storage, (b) the force applied to the body during exercise, the lower pad is made of insulating cotton and wear-resistant washed canvas (sandwich mesh).
Figure 7 illustrates the design features of the chest harness: (1) equipped the upper body of the dog with a space for light devices (batteries for smart devices, simple first aid supplies, and communication equipment) for survivor; (2) as shown in the figure $7 \mathrm{a}$, the side rings inserted from both sides and placed on the upper back. During the search, the trainer followed the dog in front, and strap is with tension; the traction point placed in the upper region to avoid neck strain. The illustration (Figure 8) shows that the force is directed to both sides through the outer strap to distribute it. The D-ring connects both sides with the lower abdominal support belt, and control belt of trainer. The force in three directions joined at chest. The overall design of the chest harness shows in the diagram (Figure 9). The abdomen are soft materials to avoid gaps and friction. When the chest harness is properly fitted to the body, the dog will not hook on the protrusions or tree trench when moving.

The flexible adjustment structure avoids gaps. The harness is soft to coordinate with the body shape. The designed strap presents lots of curvature elements. Based on the ergonomic and strap's curvature, the software using for CAD drawing is the Catia version-6.

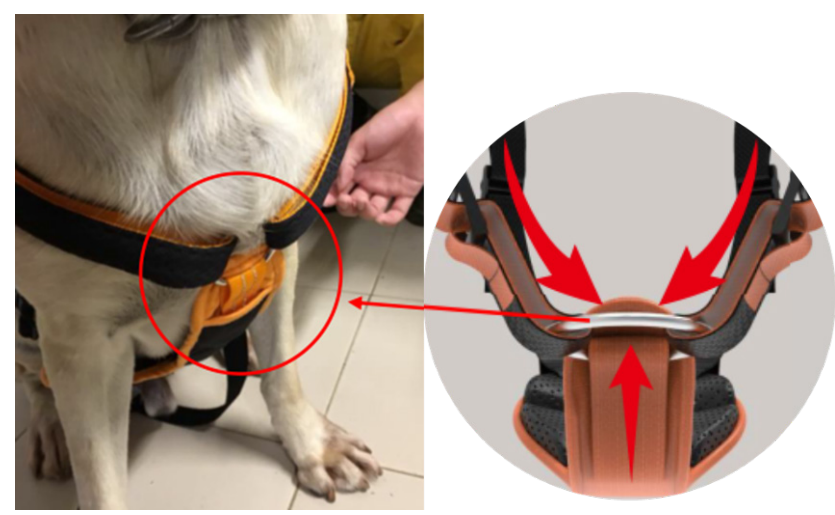

Figure 8: Combination of straps on chest

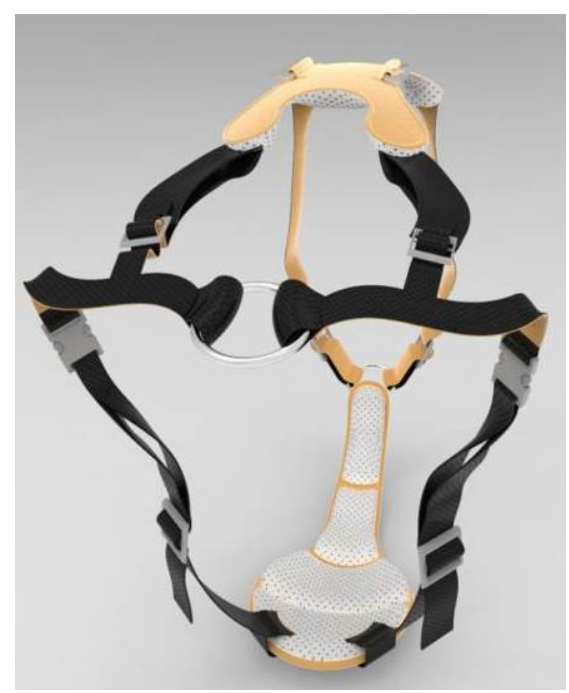

Figure 9: The 3D rendering of the chest strap

\subsection{Smart Collars}

Combined the existing wireless transmission and positioning technology, the communication between the dog and the rescuer is better in a large range search. Traditionally, dog barks to hint trainer, this touching way extended nearby areas. With wireless device, dog barks trigger the signal of the transmission position 
data and notify the trainer of the location of the victim (Figure 10a), wireless touching way extended more than $150 \mathrm{~m}$ diameter areas. The injury of the dog is a great loss of team, so their physical conditions are monitoring by trainer [23]. The sensor in the collar transmit the physical condition of dog, which allowing the trainer to adjust schedule. This function prevents the dog from being exhaustion.

The search mission is larger in the open field. The indoor WiFi router operating on the $2.4 \mathrm{GHz}$ frequency band has a radio wave of up to $50 \mathrm{~m}$, and it can be greater than $92 \mathrm{~m}$ outdoors. Based on IEEE 802.15. 4a standard, above 200 meters electromagnetic wave range can be reach with UWB [24]. User selects suitable module based on desired range. Figure 10b shows the concept of Balloon floating positioning. The UAVs generate a lot of electromagnetic waves and noise, which affect the concentration of dogs. We chose movable balloon set to place the RF module along with the balloon; hovering over the disaster area, it can quickly move to the nearby search areas. The floating balloon will move slightly with the ambient wind but does not affect the position's accuracy much.

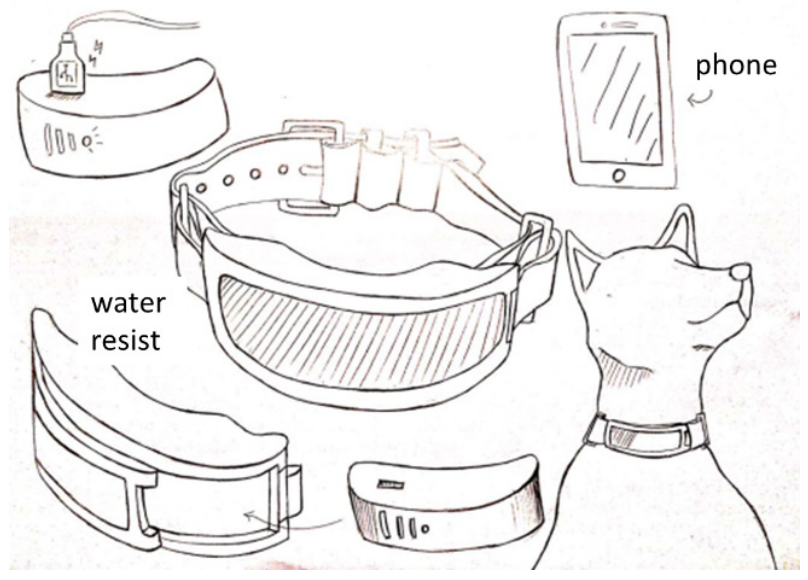

(a)

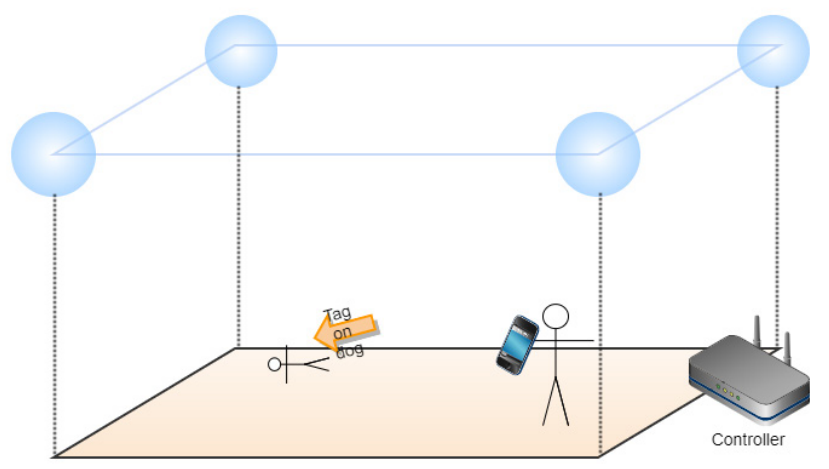

(b)

Figure 10 The proposed design: (a) smart ring module, (b) Balloon floating positioning. The UWB constructed with Semtech SX1276 Dragino LoRa Shield (with Arduino via SPI).

\subsection{Implementation}

A wireless remote connection collar was realized with Arduino platform, the system included a trainer side App to receive signal and arrange current task. The interaction between the smart collar and the rescuer's mobile phone are using Wifi signal. Since the
Arduino system is an open platform, many Shield modules are available.

Sometimes, the extended search area cannot cover by Bluetooth signal; therefore, we selected the WiFi or Utrawideband module as the wireless transmission medium. When the rescue team enters this domain, they will deploy nodes which connects with the tag on smart collars. The mobile phones also share connection and able to communicate with other devices.

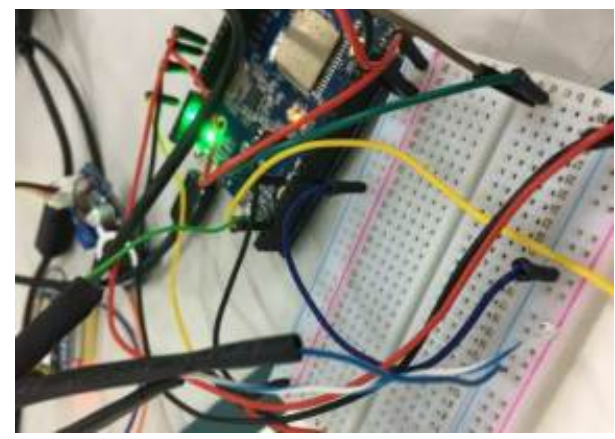

(a)

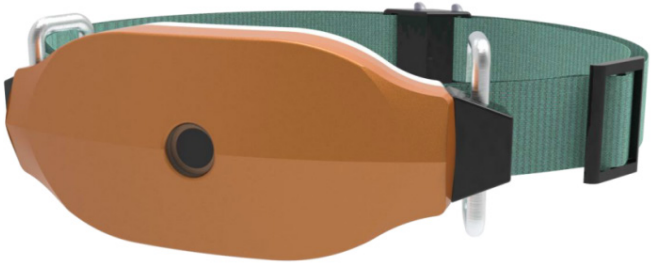

(b)

Figure 11: Components used in the circuit: (a) wireless remote connection realized with Arduino Shield (Ameba RTL8195), WiFi antenna, temperature and humidity sensor (DHT22), and sound sensor (NEO-7M-CAPP), (b) 3D printed chassis structure.

The Arduino Shield [25] used include GPS, speakers, Grove main control version. The breadboard wiring implementation shows in Figure 11a. The appearance of the smart collar (3D printed) contains miniaturized circuit board and sensors (Figure $11 \mathrm{~b}$ ); the collar placed on the neck area of the rescue dog with an adjustable belt. During the task, the collar might contact water, to maintain the stability of the circuit, so we installed a rubber O-ring placed in chassis gap for waterproofing of the circuit board.

\subsection{Software and Trainer's APP}

Heartbeat/ temperature/ and GPS status recorded by Smart collar. The squeak signal is identified with the trainer's App. The user interface can be implemented using Android APK. The dog's track record and the graphical display enhance recognition (Figure 12a). Collected dog's physical condition will display on phone screen so that the trainer can immediately prevent the dog from being exhaustion (Figure 12b).

The major Information are:

- Positioning: The dog's barks activates and informs the trainer which reveal the location of the target (Fig 12b) of the target in the mission.

- Call back: When the body temperature exceeds the set value or the task exceeds the time, the coach issues a warning. Use the withdrawal button to call the module attached to the dog and make a sound (instruction return). The training procedures 
are: Call the dog's name enthusiastically and give the command: "Come! Come!" If he walks towards you, praise it. When the dog catches up, give him some snacks and compliments immediately. After several successful exercises, try to say the word alone. The dog understands the sound in previous training when rescuers click the recall button and remotely generate the same sound signal. When the success continues, try the same action with the remote microphone. The proposed device mechanism is vocal sound, the inertia of signal/ action built in early time excites the dog's physiological reasoning.

- Guidance: rescuer asks the survivor person to prompt the dog to get the direction (Figure 13). The recorded path and target's response enabled speed up of target finding.

- Dog's background: The dog's characteristics and the training performance can access by the trainer, through specific description data helps conduct of those tasks with specific dog.

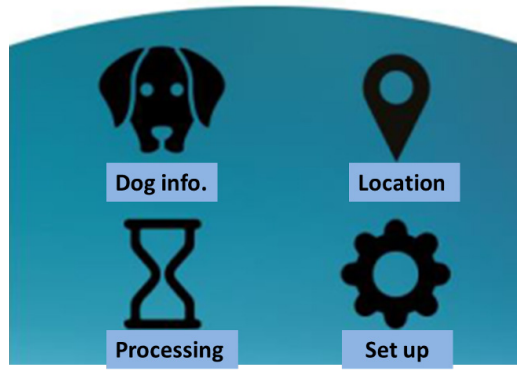

(a)

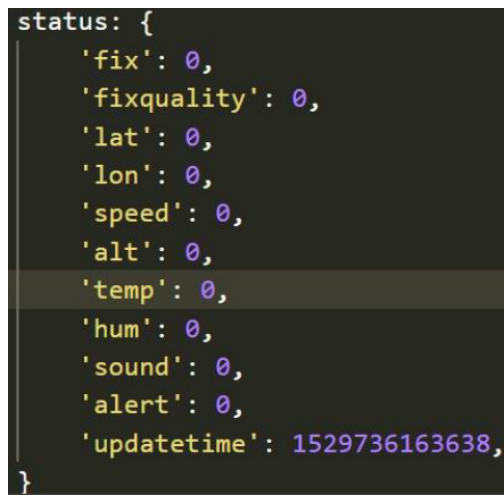

(b)

Figure 12: The main page of (a) App interfaces; and (b) code of Arduino board; where fix: successful positioning; speed: moving speed; lat/lon: latitude and longitude; Temp/Hum: temperature and humidity; Sound: volume of barking; alert: notifying back

\subsection{User survey}

Taichung established a search and rescue dog team in 2016, and had passed the Mission Readiness Test international mission rescue certification. Training require a lot of human resources including build society contact to successfully complete search tasks. Among the team, "Tie Xiong" found survivors in the rubble during the Hualien earthquake in 2018 . We took the chest strap to the training site and test it by the commander during the exercise. At the verification stage, we made a prototype with soft materials for user test including magnitude of pressure, the shear force on skin, and the loading. The normal stress around chest does decrease due to force distribute on supporting surface. Per Figure
7 and 8 , the most considerable stress occurs on the arch ribs due to pull.

The App interface is properly matched with the mobile phone, and the touch interface for quick setting and operation. The timely response is helpful for urgent handling in emergency situations. The current Arduino modules are large in size and easily interfere with the movement of the dog; in the future, this problem can be overcome through the miniaturization of a customized circuit board.

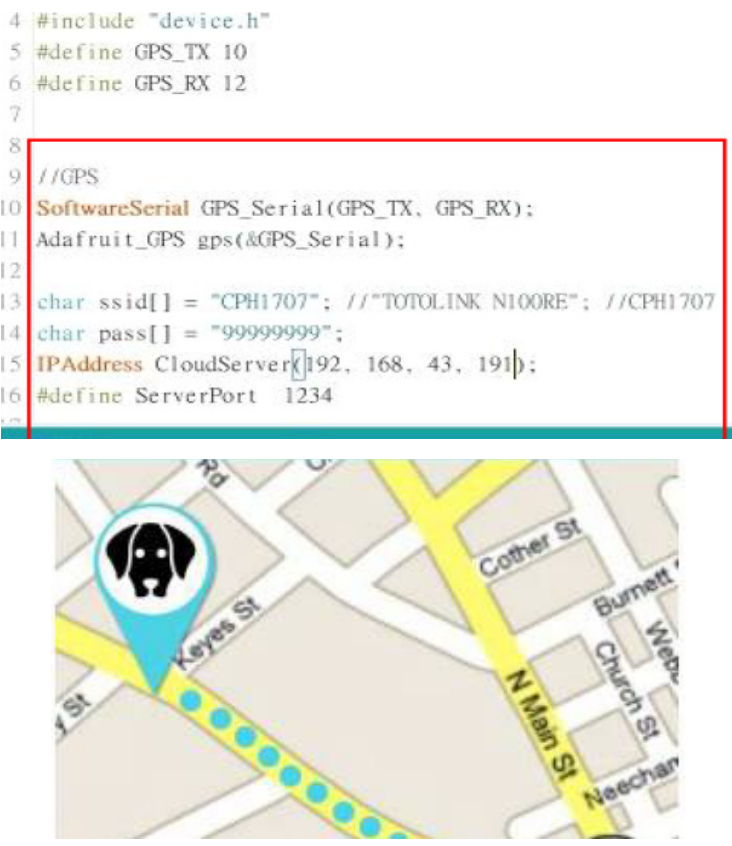

Figure 13: GPS positioning help target located on the trainer mobile App.

\section{Conclusions}

Based on situational stories and on-site observation data, we have unified the special needs in the rescue situation. The main design proposal includes a strap and a smart collar; the strap that meets the rescue dog's situation. It can be adjusted to suit individual needs. Smart sensing improves task performance; IoT chest strap helps communication and increases the efficiency of search and rescue. The smart collar records the status, and remote voice paging helps to send voice commands. Arduino's rich development modules including GPS and speakers are conducive to the prototype. Researchers found that the dog's wearable sensor improves the dog-human interaction during training.

Many technologies reported; this work looks more at user's needs through multi-disciplinary aspects of view. The conceptual situation requires the realization of the prototype to show the suitability of the concept. From the perspective of design education, we show the main parts through cross-field integration (mechanical/electronic, hardware/software). This proved that designers need to enhance cross-domain sentiment to stand at the high point of design thinking in order to propose appropriate solutions.

\section{References}

[1] L. Lit, C.A. Crawford, "Effects of training paradigms on search dog performance," Applied Animal Behaviour Science, 98(3-4), 277-292, 2006.

[2] K.E. Jones, K. Dashfield, "Search-and-rescue dogs: an overview for veterinarians," Journal of the American Veterinary Medical Association, 225(6), 854-860, 2004. 
[3] C. Zeagler, C. Byrne, G. Valentin, "Search and rescue: dog and handler collaboration through wearable and mobile interfaces" Proceedings of the Third International Conference on Animal-Computer Interaction, 6, 2016.

[4] M.W. Trevino, "Ergonomic pet harness with enhanced retractable leash" United States Patent Application US 15/398,603. 2018.

[5] S.L. Sokolowski, C. Bettencourt, "Investigation of 3D Functional grip shape to design products for dog walking and hiking," International Conference on Applied Human Factors and Ergonomics, 597-604, 2020.

[6] Y.H. Yun, "X-strap dog harness," United States Patent Application US 29/395,500. 2018.

[7] I.P. Hoskinson, "Ergonomic pet carrier for single-person use," United States Patent Application US 16/104,847, 2019.

[8] I. Greatbatch, J.G Rebecca, and S. Allen, "Quantifying search dog effectiveness in a terrestrial search and rescue environment" Wilderness \& Environmental Medicine, 26(3), 327-334, 2015.

[9] J.M. Alcaidinho, "The internet of living things: Enabling increased information flow in dog-human interactions," Ph.D. diss., Georgia Institute of Technology, 2017.

[10] A.C. Jones, R.A. Josephs, "Interspecies hormonal interactions between man and the domestic dog (Canis familiaris)," Hormones and Behavoiur, 50(3), 393-400, 2006

[11] E. Kubinyi, "Dog and owner demographic characteristics and dog personality," Behavioural Processes, 81(3), 392-401, 2009.

[12] Z. Horvàth, "Affiliative and disciplinary behaviour of human handlers during play with their dog affects concentrations in opposite directions," Hormones and Behaviour, 54, 107-114, 2008

[13] J.M. Ley, P.C. Bennett, "Understanding personality by understanding companion dogs," Anthrozoös, 20(2), 113-124, 2008.

[14] C. Mancini, S. Li, J. Valencia, D. Edwards, "Towards multispecies interaction environments: Extending accessibility to canine users," in ACM International Conference Proceeding Series, Association for Computing Machinery, 2016, doi:10.1145/2995257.2995395.

[15] F.L. Chao, "Evaluation of industrial usefulness of pet companion for elderly," Eco-design, 2013

[16] S. Mealin S, M. Foster, K. Walker, "Creating an evaluation system for future guide dogs: a case study of designing for both human and canine needs," In Proceedings of the Fourth International Conference on Animal-Computer Interaction, 1-6, 2017.

[17] Y.C. Chen, I. Alexsander, C. Lai, and R.B. Wu, "UWB-assisted highprecision positioning in a UTM prototype," 2020 IEEE Topical Conference on Wireless Sensors and Sensor Networks (WiSNeT), 42-45, 2020.

[18] L. Bruno, P. Robertson, "Wislam: Improving footslam with wifi," In 2011 International Conference on Indoor Positioning and Indoor Navigation, 1-10, 2011.

[19] M.R. Endsley, Designing for situation awareness: an approach to usercentered design.

[20] B. Ipaki, Z. Merrikhpour, "Designing bike shift lever with user-centric design approach," Journal of Ergonomics, 6(3), 43-54, 2018.

[21] S. Mazzetto, "A practical, multidisciplinary approach for assessing leadership in project management education," Journal of Applied Research in Higher Education. 11(1), 50-65, 2019.

[22] H. Gu, X. Cai, J. Zhou, F. Zhang, "A coordinate descent method for multidisciplinary design optimization of electric-powered winged UAVs," In2018 International Conference on Unmanned Aircraft Systems (ICUAS), 1189-1198, 2018.

[23] R. Brugarolas, S. Yuschak, D. Adin, L. Sherman, A. Bozkurt, " Simultaneous monitoring of canine heart rate and respiratory patterns during scent detection tasks," IEEE Sensors Journal, 19( 4), 1454-1462, 2019.

[24] M. Poturalski, J.P. Hubaux, "Distance bounding with IEEE 802.15. 4a: Attacks and countermeasures," IEEE Transactions on Wireless Communications, 10(4), 1334-1344, 2011.

[25] R.B. Brufau, "Towards automated canine training: wearable cyber-physical systems for physiological and behavioral monitoring of dogs," 2016 